\title{
Dirac operator with different potentials on edges of quantum graph: resonance asymptotics
}

\author{
A. G. Belolipetskaia, I. Y. Popov \\ ITMO University, Kronverkskiy, 49, Saint Petersburg, 197101, Russia \\ annabe11502@mail.ru,popov1955@gmail.com
}

DOI 10.17586/2220-8054-2021-12-4-425-429

\begin{abstract}
Asymptotics of resonances for the Dirac operator with different potentials on edges of a quantum graph with the Kirchhoff coupling conditions at vertices is studied. The results are obtained for a quantum graph that consists of a compact interior and a finite number of exterior edges of infinite length connected to the interior.
\end{abstract}

Keywords: quantum graph, Dirac operator, asymptotics, resonance.

Received: 18 July 2018

\section{Introduction}

In the past few decades, quantum graphs have become the subject of intense study. We will not describe in detail the history of the development of the theory of quantum graphs, but refer to works [1,2]. In this article, we will study the Weyl asymptotics of resonances for the Dirac operator on a quantum graph with Kirchhoff coupling conditions at the vertices.

As for the Weyl asymptotics of resonances, almost all results were obtained for the Schrödinger operator acting on the edges of a quantum graph. For example, E. B. Davies and A. Pushnitski in prior research [3] obtained results for a quantum graph, on the edges of which the Schrödinger operator acts, and Kirchhoff constraints are used as the connection conditions at the vertices. Earlier, a similar problem with connection conditions at vertices of a general type was also investigated, the results can be seen, for example, in the articles $[4,5]$. In addition to the above, results were also obtained by adding a magnetic field [6] to the system. We would like to obtain similar results for the Dirac operator acting on the edges of a quantum graph.

Resonances have attracted great attention over time. There are a number of works concerning the problem of resonances, particularly the completeness of resonance states which is related to the resonance asymptotics (see, e.g., [7-17]). The purpose of this paper is to investigate the asymptotics of the resonances of the Dirac operator on a quantum graph. The Dirac operator $D$ at edge $e$ has the domain $W_{2}^{1}(e) \otimes \mathbb{C}^{2}, W_{2}^{1}(e)$ is the Sobolev space. At $j-$ th edge, it acts as follows:

$$
D_{j}=-i \frac{d}{d x} \otimes \sigma_{1}+a_{j} \otimes \sigma_{3}
$$

where $\sigma_{1}=\left(\begin{array}{ll}0 & 1 \\ 1 & 0\end{array}\right), \sigma_{3}=\left(\begin{array}{cc}1 & 0 \\ 0 & -1\end{array}\right), a_{j} \in \mathbb{R}$ is some constant, specific for each edge. As the conditions for the connection at the vertices, we will use the assumption of the continuity of the function $f(1)$ and the Kirchhoff coupling conditions:

$$
\sum_{j} \partial f_{e_{j}}(V)=0
$$

where $\partial f_{e_{j}}(V)=f_{e_{j}}^{\prime}(0)$ if the vertex $V$ is the beginning of the edge $e_{j}$, and $\partial f_{e_{j}}(V)=-f_{e_{j}}^{\prime}\left(\rho\left(e_{j}\right)\right)$ if the vertex $V$ is the end of the edge $e_{j}$ and $\rho\left(e_{j}\right)$ is edge length $e_{j}$.

The main result about the asymptotics of resonances will be given for the function $N(R, F)$, which calculates the number of zeros of the function $F(k)$, taking into account their multiplicity, not exceeding the parameter $R$ :

$$
N(R, F)=\{\# k: F(k)=0 \quad \text { and } \quad|k|<R\},
$$

where the form of the function $F$ will be described below. 


\section{Preliminary}

As mentioned previously, we will consider a quantum graph consisting of the so-called inner and outer parts. The rigorous mathematical definitions for the inside and outside are taken from [3] and provided below.

Definition 2.1. An edge $e \in G$ is internal if it has finite length. Other-wise, the edge $e \in G$ is external.

Definition 2.2. A vertex $v \in G$ is interior if all edges outgoing from it are interior. Otherwise, the vertex $v \in G$ is external.

Definition 2.3. The interior of the quantum graph $G$ is the quantum graph $G_{0}$, which contains all the interior edges of the graph $G$ and the vertices that are their ends.

Definition 2.4. The volume of the inner quantum graph is equal to the sum of the lengths of all inner edges.

It will be shown that the topological structure of a quantum graph affects the form of the asymptotics of resonances. In studying the asymptotics of resonances in a similar problem with the Schrödinger operator acting on the edges, the so-called balanced vertices play an important role. If the Dirac operator acts on the edges, then the balanced vertices also play an important role.

Definition 2.5. An outer vertex $v \in G$ is balanced if the number of inner and outer edges going out from it is equal. Otherwise, the vertex $v \in G$ is unbalanced.

Let us study in more detail the form of the system of differential equations (1) and coupling equations at the vertices (2). On each interior edge, the solution to the differential equation (1) is the following vector-function:

$$
y_{j}(x)=b_{j} e^{i\left(\lambda-a_{j}\right) x}+c_{j} e^{-i\left(\lambda-a_{j}\right) x},
$$

and on each outer edge the solution (1) is the following function:

$$
y_{j}(x)=d_{j} e^{i\left(\lambda-a_{j}\right) x} .
$$

Strictly speaking, on the outer edge, the solution (1) has the form $y_{j}(x)=d_{j} e^{i\left(\lambda-a_{j}\right) x}+\tilde{d}_{j} e^{-i\left(\lambda-a_{j}\right) x}$, but within the framework of this problem, we assume that the boundary conditions at infinity of the outer edge are such that $\tilde{d}_{j}=0$ for any outside edge.

When describing the formulation of the problem, we used the coupling conditions at the vertices described by the equations (2). The system of coupling conditions at the vertices can be written in matrix form:

$$
A \cdot \varphi_{A}=0,
$$

where $\varphi_{A}$ is a vector whose coordinates are the variables $b_{j}, c_{j}, d_{j}$ and the values of the function $f$ at the vertices are $f\left(V_{j}\right)$.

It is easy to see that the matrix $A$ is determined ambiguously for a fixed quantum graph, namely, it is possible to change the order of the variables $b_{j}, c_{j}, d_{j}, f\left(V_{j}\right)$, as well as to change the order of the constraint equations at the vertices. The following function will be taken as the function $F$, which will be used to study the asymptotics of resonances (3):

$$
F(\lambda)=\operatorname{det}(A),
$$

where the matrix $A$ can be any matrix describing the conditions of communication at the vertices of a quantum graph (6). This definition is correct, since the function is the determinant of the invariant up to a sign with respect to the permutation of rows and columns. In what follows, as a matrix $A$, it is more convenient to use the matrices $A^{+}, A^{-}$:

$$
\begin{aligned}
& A^{+} \cdot \varphi^{+}=0, \\
& A^{-} \cdot \varphi^{-}=0 .
\end{aligned}
$$

In order to describe the form of the vectors $\varphi^{ \pm}$and matrices $A^{ \pm}$, we introduce auxiliary notation. Consider some vertex $V_{i}$. Suppose that it contains $p$ interior edges $e_{i j}$, the solutions of which are characterized by the coefficients $b_{i j}, c_{i j}$ (4). Suppose $q$ of the inner edges $e_{i k}$ emerges from the vertex, the solutions of which are characterized by the coefficients $\tilde{b}_{i k}, \tilde{c}_{i k}$ (4). And finally, suppose that $r$ of outer edges $e_{i l}$ emerge from the vertex $V_{i}$, the solutions of which are characterized by the coefficients $d_{i l}$ (5). Then the vectors $\varphi^{ \pm}$are equal to the following:

$$
\varphi^{ \pm}=\left(V_{1}^{ \pm}, \ldots, V_{N}^{ \pm}\right)^{T},
$$

where $N$ is the number of vertices in a quantum graph and $V_{i}^{ \pm}$denotes the following:

$$
\begin{aligned}
V_{i}^{+} & =\left(b_{i 1}, \ldots, b_{i p}, \tilde{c}_{i 1}, \ldots, \tilde{c}_{i q}, d_{i 1}, \ldots, d_{i r}, f\left(V_{i}\right)\right), \\
V_{i}^{-} & =\left(c_{i 1}, \ldots, c_{i p}, \tilde{b}_{i 1}, \ldots, \tilde{b}_{i q}, d_{i 1}, \ldots, d_{i r}, f\left(V_{i}\right)\right) .
\end{aligned}
$$


Thus, we have decided on the order of the columns of the matrices $A^{ \pm}$, it remains to choose a convenient order of the rows. The rows of the matrix are arranged as follows. Using the notation introduced above, suppose that the vertex $V_{i}$ has the number of internal edges that enter it, is equal to $p_{i}$, the number of internal edges that go out of it is $q_{i}$, the number of external edges that go out of it, is equal to $r_{i}$. Then the first $p_{1}+q_{1}+r_{1}$ rows of the matrices $A^{ \pm}$describe the equations characterizing the continuity of the function $f$ at the vertex $V_{1}$. The next line describes the Kirchhoff constraint equation at the vertex $V_{1}$. In a similar way, $p_{2}+q_{2}+r_{2}+1$ lines describe the constraint equations at the vertex $V_{2}$, and so on for all other vertices.

It is worth noting that, depending on the choice of the orientation of the inner edges of the quantum graph, the form of the matrices $A$, and as a consequence of the matrices $A^{ \pm}$, will change. In this regard, the question arises whether the orientation of the zeros of the function $F(\lambda)$ will not be affected. It was shown in the [3] article that changing the orientation will not affect the zeros of $F(\lambda)$. And although this was proved for quantum graphs on whose edges the Schrödinger operator acts, we can also use this result, since the general form of the equation (4) coincides with the general form of the solution on the edges for the Schrödinger operator.

When investigating the asymptotics of resonances, we will use a theorem formulated below. A rigorous mathematical proof of this theorem can be found in [5].

Theorem 2.6. Let $F(k)=\sum_{r=0}^{n} k^{v_{r}} a_{r}(k) e^{i k \sigma_{r}}$, where $v_{r} \in \mathbb{R}, a_{r}(k)$ are rational functions of the complex variable $k$ with complex coefficients that do not vanish identically, and $\sigma_{r} \in \mathbb{R}, \sigma_{0}<\sigma_{1}<\ldots<\sigma_{n}$. Suppose also that $v_{r}$ are chosen so that $\lim _{k \rightarrow \infty} a_{r}(k)=\alpha_{r}$ is finite and non-zero for all $r$. There exists a compact set $\Omega \subset \mathbb{C}$, real numbers $m_{r}$ and positive $K_{r}, r=1, \ldots, n$ such that the zeros of $F(k)$ outside $\Sigma$ lie in one of $n$ logarithmic strips, each one bounded between the curves $-\operatorname{Im} k+m_{r} \log |k|= \pm K_{r}$. The counting function behaves in the limit $R \rightarrow \infty$ as:

$$
N(R, F)=\frac{\sigma_{n}-\sigma_{0}}{\pi} R+O(1)
$$

\section{Results}

Before proceeding to the main results, we prove some auxiliary statements.

Lemma 3.1. The following relation takes place:

$$
\left|\begin{array}{ccccc}
a_{1} & 0 & \cdots & 0 & -1 \\
0 & a_{2} & \cdots & 0 & -1 \\
\cdots & \cdots & \ddots & \cdots & \vdots \\
0 & \cdots & 0 & a_{n} & -1 \\
b_{1} & b_{2} & \cdots & b_{n} & 0
\end{array}\right|=\prod_{i=1}^{n} a_{i} \cdot \sum_{j=1}^{n} \frac{b_{j}}{a_{j}}
$$

To prove this, it is sufficient to add all $\left(j-\right.$ th) columns multiplied by $\frac{1}{a_{j}}$ to the last column. As a result, one obtains the triangular determinant.

As you can see from the theorem 2.6, the coefficients before $e$ in various powers of the function $F(\lambda)$ are of particular interest. For further convenience, we introduce several auxiliary variables:

$$
\begin{gathered}
e^{+}=\prod_{j=1}^{M} z_{j}, \\
e^{-}=\prod_{\substack{j=1 \\
z_{j}}}^{M} z_{j}^{-1}, \\
z^{i\left(\lambda-a_{j}\right) \rho\left(e_{j}\right)},
\end{gathered}
$$

where $\rho\left(e_{j}\right)$ is the length of the edge $e_{j}, M$ is the number of interior edges in the quantum graph. Next, we will calculate the coefficient in front of $e^{ \pm}$of the function $F(\lambda)$.

Lemma 3.2. The coefficient $c^{+}$before $e^{+}$of the function $F(\lambda)=\operatorname{det}\left(A^{+}\right)$is as follows:

$$
c_{V_{i}}=\sum_{V_{i} \in e_{j}} a_{j}-\sum_{V_{i} \in e_{k}}^{N} a_{k}+\lambda \cdot l,
$$


where $e_{j}$ are inner edges, $e_{k}$ are outer edges, $l$ is a number equal to the difference between the number of outer and inner edges containing the vertex $V_{i}$.

Proof. According to the choice of the order of rows and columns for the matrix $A^{+}$, it has the following form:

$$
A^{+}=\left(\begin{array}{cccc}
\tilde{V}_{1} & \ldots & \cdots & \cdots \\
\cdots & \tilde{V}_{2} & \cdots & \cdots \\
\vdots & \vdots & \ddots & \vdots \\
\cdots & \cdots & \cdots & \tilde{V}_{N}
\end{array}\right)
$$

where the blocks $\tilde{V}_{i}$ characterize the vertex $V_{i}$, have the size $\left(p_{i}+q_{i}+r_{i}+1\right) \times\left(p_{i}+q_{i}+r_{i}+1\right)$, where the designations $p_{i}, q_{i}, r_{i}$ are taken from the description of the construction of the matrix $A^{+}$, and contain the elements $z_{j}=e^{i\left(\lambda-a_{j}\right) \rho\left(e_{j}\right)}$. Moreover, by the construction of the matrix $A^{+}$, the elements of $z_{j}$ are contained only in the blocks $\tilde{V}_{j}$. Then, it is easy to see that the coefficient before $e^{+}$in $\operatorname{det}\left(A^{+}\right)$is the same as the coefficients before $e^{+}$of the function $F_{V}$, which is equal to the following:

$$
F_{V}=\prod_{i=1}^{N} \operatorname{det} \tilde{V}_{i}
$$

Recalling the description of the matrix $A^{+}$, as well as the system of differential equations (4), (5) with matching conditions at the vertices (2), let us study in more detail the form of the block $\tilde{V}_{i}$ :

$$
\left(\begin{array}{cccccccccc}
z_{i 1} & 0 & \ldots & \ldots & \ldots & \ldots & \ldots & \ldots & 0 & -1 \\
\ldots & \ddots & \ldots & \ldots & \ldots & \ldots & \ldots & \ldots & \ldots & \ldots \\
\ldots & \ldots & z_{i p} & 0 & \ldots & \ldots & \ldots & \ldots & 0 & -1 \\
0 & \ldots & 0 & 1 & 0 & \ldots & \ldots & \ldots & 0 & -1 \\
\ldots & \ldots & \ldots & \ldots & \ddots & \ldots & \ldots & \ldots & \ldots & \ldots \\
0 & \ldots & \ldots & \ldots & 0 & 1 & 0 & \ldots & 0 & -1 \\
0 & \ldots & \ldots & \ldots & \ldots & 0 & 1 & \ldots & \ldots & -1 \\
\ldots & \ldots & \ldots & \ldots & \ldots & \ldots & \ldots & \ddots & \ldots & \ldots \\
0 & \ldots & \ldots & \ldots & \ldots & \ldots & \ldots & 0 & 1 & -1 \\
-z_{i 1} \chi_{i 1} & \ldots & -z_{i p} \chi_{i p} & -\chi_{j 1} & \ldots & \left.-\chi_{j q}\right) & \chi_{k 1} & \ldots & \chi_{k r} & 0
\end{array}\right),
$$

where $\chi_{i j}=\lambda-a_{i j}$, the vertex $V_{i}$ has $p$ inner edges entering it, $q$ inner edges leaving it, and $r$ outer edges leaving it. Using lemma 3.1, we calculate $\operatorname{det}\left(\tilde{V}_{i}\right)$ :

$$
\begin{gathered}
\operatorname{det}\left(\tilde{V}_{i}\right)=\prod_{l=1}^{p} z_{i l} \cdot\left(\sum_{l=1}^{p}\left(-\lambda+a_{i l}\right)+\sum_{m=1}^{q}\left(-\lambda+a_{j m}\right)+\sum_{n=1}^{r}\left(\lambda-a_{k n}\right)\right)= \\
=\prod_{l=1}^{p} z_{i l} \cdot\left(\sum_{e_{j} \in G_{0}} a_{j}-\sum_{e_{k} \in G \backslash G_{0}} a_{k}+\lambda \cdot(r-p-q)\right)=\prod_{l=1}^{p} z_{i l} \cdot c_{V_{i}} .
\end{gathered}
$$

After calculating $\operatorname{det}\left(\tilde{V}_{i}\right)$, it is easy to determine what $F_{V}$ is equal to:

$$
F_{V}=\prod_{i=1}^{M} z_{i} \cdot c_{V}
$$

where $M$ is equal to the number of interior edges. From 18 it can be seen that $c^{+}=\prod_{i=1}^{N} c_{V}$. Thus, the lemma is proved.

Note. It is worth mentioning similar coefficients for $e^{-}$. If solutions of the form $\tilde{d}_{i} e^{-i\left(\lambda-a_{i}\right) x}$ are considered on the outer edges, the coeffici-ents will be similar to the results of the lemma 3.2 up to sign. In the case of considering solutions of the form $d_{i} e^{i\left(\lambda-a_{i}\right) x}$ on the outer edges, the coefficients will be similar to the results of the lemma 3.2 with the only difference that the sums over the outer and inner edges will not be subtracted, but added.

Now let us formulate a theorem, which is the main result of this article. 
Theorem 3.3. Consider a quantum graph consisting of a compact interior and a finite number of edges of infinite length that are attac-hed to the interior of the quantum graph. The edges of this quantum graph will be acted upon by the Dirac operator 1. As the connection conditions at the vertices of the quantum graph, we will use the Kirchhoff connection conditions and the assumption of the continuity of the solution function on the quantum graph. Then the asymptotics of the resonance counting function as $R \rightarrow \infty$ is of the form:

$$
N(R, F)=\frac{2 W}{\pi} R+O(1)
$$

where the variable $W$ satisfies the following inequalities:

$$
0 \leq W \leq V=\sum_{j=1}^{N} l_{j}
$$

It should be noted that $W<V$ is equivalent to the existence of an external balanced vertex $V_{i}$, in which the following relation holds:

$$
\sum_{V_{i} \in e_{j}} a_{j}=\sum_{V_{i} \in e_{k}} a_{k}
$$

Proof. To prove the statement (20), we use the theorem 2.6. Then, using the notation from this theorem 2.6 and using the results of the lemma 3.2, we obtain $-V \leq \sigma_{0} \leq 0,0 \leq \sigma_{n} \leq V$, where $V=\sum_{j=1}^{N} l_{j}$. Consequently $0 \leq \sigma_{n}-\sigma_{0} \leq 2 V$ and by theorem $2.6 N(R, F)=\frac{2 W}{\pi} R+O(1)$, where $0 \leq W \leq V$, as required in the relation (20).

To prove the relation (21), note that for the inequality $W<V$ to hold, it is necessary and sufficient that at least one of the two inequalities $-V<\sigma_{0}$ or $\sigma_{n}<V$. That is, the coefficient before $e^{+}$or $e^{-}$must be equal to 0 . In lemma 3.2 the coefficient before $e^{+}$of $F(\lambda)$ was determined. It is easy to see that the coefficient before $e^{+}$is 0 if and only if there is an external balanced vertex for which the relation (21) holds.

Thus, the theorem is proved.

\section{References}

[1] Berkolaiko G., Kuchment P. Introduction to quantum graphs. American Mathematical Soc., 2013.

[2] Exner P. et al. (ed.). Analysis on Graphs and Its Applications: Isaac Newton Institute for Mathematical Sciences, Cambridge, UK, January 8-June 29, 2007. American Mathematical Soc., 2008.

[3] Davies E.B., Pushnitski A. Non-Weyl resonance asymptotics for quantum graphs. Analysis and PDE, 2011, 4, P. $729-756$.

[4] Exner P., Lipovský J. Resonances from perturbations of quantum graphs with rationally related edges. Journal of Physics A: Mathematical and Theoretical, 2010, 43, P. 105301.

[5] Davies E.B., Exner P., Lipovsky J. Non-Weyl asymptotics for quantum graphs with general coupling conditions. J. Phys. A: Math. Theor., 2010, 43, P. 474013.

[6] Exner P., Lipovsky J. Non-Weyl resonance asymptotics for quantum graphs in a magnetic field. Phys. Lett. A, 2011,375 , P. $805-807$.

[7] Aslanyan A., Parnovski L., Vassiliev D. Complex resonances in acoustic waveguides. Q. J. Mech. Appl. Math., $2000,53(3)$, P. $429-447$.

[8] Blinova I.V., Popov I.Y. Quantum graph with the Dirac operator and resonance states completeness. Operator Theory: Adv. Appl., 2018, 268, P. 111-124.

[9] Christiansen T. Some upper bounds on the number of resonances for manifolds with infinite cylindrical ends. Ann. Henri Poincare, 2002, 3, P. 895-920.

[10] Edward J. On the resonances of the Laplacian on waveguides. J. Math. Anal. Appl., 2002, 272, P. 89-116.

[11] Gerasimov D.A., Popov I.Y. Completeness of resonance states for quantum graph with two semi-infinite edges. Compl. Var. Elliptic Eq., 2018, 63, P. 996-1010.

[12] Popov I.Y., Blinova I.V. Quantum graph in a magnetic field and resonance states completeness. Operator Theory: Adv. Appl., 2020, 276, P. 540-553.

[13] Sjöstrand J.,Zworski M. Complex scaling and the distribution of scattering poles. J.Amer. Math. Soc., 1991, 4, P. 729-769.

[14] Blinova I.V., Popov I.Y., Popov A.I. Resonance states completeness for relativistic particle on a sphere with two semi-infinite lines attached. Journal of King Saud University - Science, 2020, 32(1), P. 836-841.

[15] Popov I.Y., Popov A.I. Quantum Dot with Attached Wires: Resonant States Completeness. Reports on Mathematical Physics, 2017, 80(1), P. $1-10$.

[16] Popov I., Gerasimov D., Blinova I., Popov A. Incompleteness of resonance states for quantum ring with two semi-infinite edges. Analysis and Mathematical Physics, 2019, 9(3), P. 1287-1302.

[17] Duclos P., Exner P., Meller B. Open quantum dots: Resonances from perturbed symmetry and bound states in strong magnetic fields. Rep. Math. Phys., 2001, 47(2), P. 253-267. 